\title{
Association between coffee consumption and risk of bladder cancer in a meta- analysis of 16 prospective studies
}

\author{
Zhi-Wei Dai ${ }^{1}$, Ke-Dan Cai ${ }^{1}$, Fu-Rong Li ${ }^{2}$, Xian-Bo Wu ${ }^{2}$ and Guo-Chong Chen ${ }^{3^{*}}$ (D)
}

\begin{abstract}
Background: Current evidence remains equivocal as to whether and how consumption of coffee may be associated with risk of bladder cancer, and potential influence of confounding by smoking on this association is yet to be elucidated. We conducted an updated meta-analysis of prospective studies to address these issues.

Methods: Relevant studies were identified by searching PubMed and EMBASE databases from inception to April 2019. A random-effects model was used to estimate summary relative risk (RR) with corresponding 95\% confidence interval (Cl) of bladder cancer associated with coffee consumption.

Results: The final analysis included 16 prospective studies comprising 2,122,816 participants and 11,848 bladder cancer cases. Overall, coffee consumption was not associated with risk of bladder cancer (RR high-vs-low $=1.07$, 95\% Cl: 0.96-1.20). The lack of association persisted in the strata defined by sex or participants' smoking status. Meta-regression analyses identified the number cases $(P$ difference $=0.06)$ and the degree of adjustment for smoking $(P$ difference $=0.04)$ as potential sources of heterogeneity. There was an increased risk of bladder cancer related to higher coffee consumption among studies with fewer cases ( $R R$ high-vs-low $=1.38,95 \% \mathrm{Cl}$ : $1.05-1.81$ ) and among those with poorer adjustment for smoking (RR high-vs-low $=1.48,95 \% \mathrm{Cl}$ : 1.14-1.93). Results were similar in the dose-response analyses ( $\mathrm{RR}_{1}$ cup/d $=1.01,95 \% \mathrm{Cl}$ : 0.98-1.03).

Conclusion: Best evidence available to date does not support an independent association between coffee consumption and bladder cancer risk. Some direct associations observed in individual studies may be a result of residual confounding by smoking.
\end{abstract}

Keywords: Coffee, Bladder cancer, Cohort studies, Meta-analysis

\section{Background}

Coffee is among the most commonly consumed beverages worldwide. As such, a small impact of coffee drinking on health risk could lead to significant public health consequences. Over the past decades, many epidemiologic studies have been carried out to extensively evaluate potential influences coffee drinking on multiple health outcomes, including the developments of various types of cancer [1]. While inverse associations between coffee consumption and risk of cancers at specific sites including liver and endometrium have been documented [2], some evidence for an

\footnotetext{
* Correspondence: Guochong.Chen@einstein.yu.edu

${ }^{3}$ Department of Epidemiology and Population Health, Albert Einstein College of Medicine, 1300 Morris Park Avenue, Bronx, NY 10461, USA

Full list of author information is available at the end of the article
}

increased risk of bladder cancer related to coffee drinking also emerged. In 1991, the International Agency for Research on Cancer (IARC) Monographs classified coffee as "possibly carcinogenic" to the bladder [3]. The IARC Monographs reviewed the accumulative evidence on coffee and cancer in 2016 and updated the statement that "overall coffee drinking was evaluated as unclassifiable as to its carcinogenicity to humans" [4]. Nevertheless, the Monographs also called for additional large prospective studies carefully accounting for important confounders, especially cigarettes smoking that is often correlated strongly with coffee drinking, for more definite conclusions.

Since the release of the 2016 statement, five large prospective studies [5-9] examining the association between coffee consumption and risk of bladder cancer have been

(c) The Author(s). 2019 Open Access This article is distributed under the terms of the Creative Commons Attribution 4.0 International License (http://creativecommons.org/licenses/by/4.0/), which permits unrestricted use, distribution, and 
published, comprising more than 1.45 million participants and 9000 bladder cancer cases. More importantly, four [5-8] of the five studies additionally examined the association among never-smoking participants. These data allow for an independent evaluation of coffee-bladder cancer association among never smokers to completely avoid residual confounding by smoking. Therefore, we performed a large updated meta-analysis to assess the association between coffee consumption and risk of bladder cancer.

\section{Methods}

\section{Literature search}

We performed a literature search in PubMed and EMBASE databases for potentially relevant studies published from inception to April 2019. We used the search term "coffee" in combination with "bladder cancer", "urinary cancer", or "urothelial carcinoma", without imposing language restrictions. The detailed search strategies are reported in Additional file 1. To identify any additional studies, we also hand searched the bibliographies of retrieved full publications and those of several previous meta-analyses of coffee consumption and risk of bladder cancer [10-13]. The eligibility of the identified records was accessed independently by two authors. Any disagreement between the authors was resolved by group discussion.

\section{Study selection}

Potentially eligible studies were prospective studies (i.e. prospective cohort, case-cohort, or nested case-control studies for which consumption of coffee were recorded prior to cancer diagnosis) that evaluated the relationship between coffee consumption and risk of incident or fatal bladder cancer. To be included in the meta-analysis, studies also had to report risk estimates such as hazard ratio, relative risks (RR), or odds ratio with corresponding 95\% confidence intervals (CI) of bladder cancer associated with coffee consumption, or report relevant data for deriving these estimates. We did not exclude studies that had small sample sizes or poor adjustments for potential confounders; instead, we evaluated the impacts of these study characteristics in predefined subgroup analyses.

\section{Data extraction}

Using a standardized data-collection form, we extracted the following characteristics from each included study: the first author's last name, publication year, country, sources of study population, years of follow-up, number of participants, age and sex of participants, the proportion of current smokers among participants, number of bladder cancer cases, and proportion of male cases. We further extracted information on different categories of coffee consumption and the corresponding fully adjusted risk estimates with 95\% CIs (by smoking status, if available), as well as adjustments for potential confounders in the statistical models. Because the association between coffee consumption and risk of bladder cancer could be particularly prone to confounding by cigarette smoking, we extracted detailed information on how smoking habits were adjusted for in the analyses (e.g. smoking status, frequency, and duration). Data were extracted by one author (Z-WD) and independently verified by other two authors (K-DC and F-RL), with any disagreement resolved by group discussions.

\section{Statistical analysis}

Given the substantial differences in the levels of coffee consumption among individual studies, we calculated both summary risk estimates of bladder cancer for the extreme categories (i.e. highest vs. lowest) and for each 1 cup/d increment of coffee consumption. We combined study-specific risk estimates using a random-effects model to account for both within- and between-study variations [14]. For studies [15-17] in which only sexspecific rather than overall results were reported, we pooled the sex-specific estimates for each study using a fixed-effect model and included the combined results in the main analyses to maintain the correct degrees of freedom for tests of heterogeneity. For one study [16] in which the reference group was not the lowest coffee consumption, and we set the lowest category as the new reference category and calculated new risk estimates according to the method of Hamling et al [18]. For two studies $[15,19]$ in which some RRs of bladder cancer were reported without $95 \%$ CIs, the CIs were also derived using the Hamling et al's methods.

If dose-response relationships between coffee consumption (e.g. 1 cup/d) and risk of bladder cancer was not examined in the original studies, we calculated the estimates using the generalized least squares trend estimation [20]. Accordingly, the levels of coffee consumption, the distributions of cases and person-years, as well as risk estimates of bladder cancer with corresponding 95\% CIs for each category of coffee consumption were extracted for the estimation of a dose-response association. For a European study [21] in which coffee consumption was expressed in daily volume $(\mathrm{ml} / \mathrm{d})$ rather than cups, we rescaled the consumption into cup/d by using $125 \mathrm{ml}$ as a standard size of a cup (validated in another European study [16]). When the number of cases or person-years in each coffee category was not reported, we estimated the distributions from total number of cases or person-years. The median or mean consumption in each category was used as the average amount, and when these values were not reported, the midpoint of the 
upper and lower boundaries was considered average amount of consumption. If the highest or lowest category was open-ended, we assumed the width of the interval to be the same as in the closest category. The results of linear dose-response analyses are presented for a $1 \mathrm{cup} / \mathrm{d}$ increment of coffee consumption. We examined potential nonlinear relationships between coffee consumption and risk of bladder cancer using restricted cubic splines, modeling three knots at percentiles 10,50 and $95 \%$ of the distribution of coffee consumption [22]. A $P$ value for nonlinearity was calculated by testing the null hypothesis that the coefficient of the second spline is equal to zero.

To explore potential sources of heterogeneity, we conducted stratified and meta-regression analyses according to the following study and population characteristics: geographic region, duration of follow-up, number of participants, sex of participants, percentage of current smoker among the study population, number of bladder cancer cases, percentage of male cases, smoking status, and adjustment for potential confounders. To better capture the different degree of being subject to confounding by smoking across individual studies, we categorized the adjustments for cigarettes smoking into three groups including: poorer adjustment for smoking: no adjustment for smoking or adjustment for smoking status only; moderate adjustment for smoking: adjustment for smoking status in addition smoking frequency (e.g. cigarettes smoked per day, or pack-years of smoking) either continuously or categorically; better adjustment for smoking: adjustment for smoking status, smoking frequency, in addition to smoking duration or lifetime smoking intensity.

Heterogeneity among studies was assessed using $\mathrm{I}^{2}$ statistics [23], with a value of $<25 \%, 25-50$ and $>50 \%$ indicating little or no, moderate, and high heterogeneity, respectively. Potential publication bias was assessed using both Begg rank correlation test and Egger linear regression test [24, 25]. All statistical analyses were carried out using STATA version 12.0 (STATA Corp., College Station, TX, USA). All $P$ values were two-sided, and the level of significance was at $<0.05$, unless explicitly stated.

\section{Results}

Study selection

A flow chart of study selection is reported in Fig. 1. Briefly, a total of 329 independent reports were identified after removing overlapping records, of which 26 were retrieved for more detailed evaluations. Three additional reports were found by screening the references of relevant publications. Thirteen of the 29 reports were excluded after reviewing the full texts. As a result, 16 publications [5-9, 15-17, 19, 21, 26-31] including 16 prospective studies were included in the meta-analysis (Sugiyama et al [7] and Lukic et al [8] both combined two independent cohorts, and we counted both as one study because no cohort-specific results were presented). Overall, the 16 prospective studies included 2,122,816 participants and 11,848 bladder cancer cases.

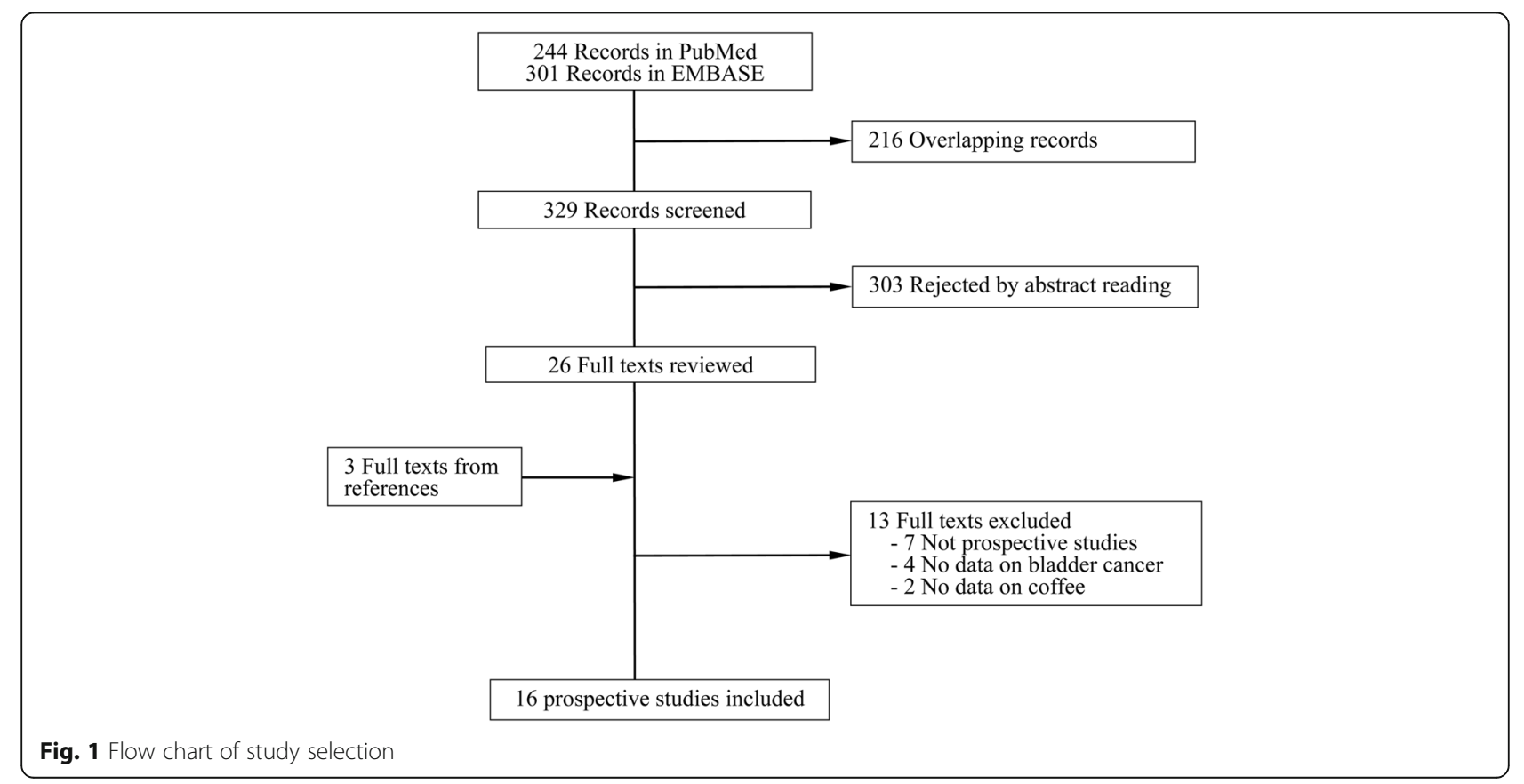




\section{Study characteristics}

The characteristics of the included studies are summarized in Table 1. Of the 16 studies, seven were from the US, six were from Europe, and three from Japan. Duration of follow up (5.3 to $23.4 \mathrm{yr}$ ), number of participants (3113 to 696,391 ), and number of cases (52 to 6012) ranged substantially across individual studies. Three studies included men only (one study [9] included totally male smokers), one study included women only, and the remaining studies recruited participants of both sexes. All but one study [5] used bladder cancer incidence as the study outcome. For the study [5] that focused on cancer mortality, only results for nonsmoking participants (former and/or never smokers) were reported. Thus, proportion of current smokers ranged from 0 to $100 \%$ among participants of the included studies. Reported results, statistical adjustments for potential confounders, as well as detailed information on adjustments for smoking behaviors for the included studies are summarized in Table 2. Among the 16 studies, three, eight, and five studies were deemed to have poorer, moderate, and better adjustments for cigarettes smoking, respectively.

\section{Meta-analysis}

A meta-analysis of the 16 prospective studies yielded a summary RR of 1.07 (95\% CI: 0.96-1.20) for the highest compared with the lowest categories of coffee consumption (Fig. 2a). One study [19] did not provide eligible data for the dose-response analysis. A dose-response meta-analysis of the remaining 15 studies showed a summary RR of 1.01 (95\% CI: 0.98-1.03) for each $1 \mathrm{cup} / \mathrm{d}$ increment of coffee consumption (Fig. 2b). Moderate heterogeneity was

Table 1 Characteristics of prospective studies included in the meta-analysis

\begin{tabular}{|c|c|c|c|c|c|c|c|}
\hline \multirow[t]{2}{*}{ Author, years } & \multirow[t]{2}{*}{ Country } & \multirow[t]{2}{*}{ Population } & \multirow[t]{2}{*}{ Duration, yr } & \multicolumn{3}{|l|}{ Participants } & \multirow{2}{*}{$\begin{array}{l}\mathrm{N} \text { of cases }(\% \\
\mathrm{M})\end{array}$} \\
\hline & & & & $\mathrm{N}$ and sex & Age, yr & $\%$ Smoker & \\
\hline $\begin{array}{l}\text { Jacobsen, } \\
1986 \text { [19] }\end{array}$ & Norway & $\begin{array}{l}\text { Norwegian cohort of } \\
\text { mostly men }\end{array}$ & 11.5 & $13,664 M ; 2891 \mathrm{~W}$ & $\geq 35$ & $\begin{array}{l}37.9 \%(\mathrm{M} \\
\text { only) }\end{array}$ & $94(69.1 \%)$ \\
\hline Mills, 1991 [26] & US & $\mathrm{AHS}$ & 5.3 & $34,198 \mathrm{M} \& W$ & $\geq 25$ & $1.4 \%$ & $52(69.2 \%)$ \\
\hline $\begin{array}{l}\text { Chyou, } 1993 \\
{[27]}\end{array}$ & US & Japanese-American men & 22.0 & 7995 M & $N R$ & $43.7 \%$ & $96(100 \%)$ \\
\hline $\begin{array}{l}\text { Stensvold, } \\
1994[15]\end{array}$ & Norway & $\begin{array}{l}\text { CVD screening } \\
\text { Participants }\end{array}$ & 10.1 & $21,735 \mathrm{M} ; 21,238 \mathrm{~W}$ & $35-54$ & $\begin{array}{l}46.1 \%(M) \\
34.1 \%(\mathrm{~W})\end{array}$ & $53(75.5 \%)$ \\
\hline $\begin{array}{l}\text { Michaud, } 1999 \\
\text { [28] }\end{array}$ & US & HPFS & 9.1 & $47,909 \mathrm{M}$ & $40-75$ & $9.6 \%$ & $252(100 \%)$ \\
\hline $\begin{array}{l}\text { Nagano, } 2000 \\
{[29]}\end{array}$ & Japan & LSS & 11.7 & $14,873 \mathrm{M} ; 23,667 \mathrm{~W}$ & $\begin{array}{l}52.8(\mathrm{M}) \\
56.8(\mathrm{~W})\end{array}$ & $35.0 \%$ & $114(72.8 \%)$ \\
\hline $\begin{array}{l}\text { Zeegers, } 2001 \\
{[16]}\end{array}$ & $\begin{array}{l}\text { The } \\
\text { Netherlands }\end{array}$ & NCS & 6.1 & $\begin{array}{l}1515 \mathrm{M} ; 1598 \mathrm{~W} \\
\text { (sub-cohort) }\end{array}$ & $55-69$ & $\begin{array}{l}34.0 \%(M) \\
20.0 \%(W)\end{array}$ & $569(93.5 \%)$ \\
\hline $\begin{array}{l}\text { Tripathi, } 2002 \\
\text { [30] }\end{array}$ & US & IWHS & 13.0 & $37,459 \mathrm{~W}$ & $55-69$ & $15.0 \%$ & $112(0 \%)$ \\
\hline $\begin{array}{l}\text { Kurahashi, } \\
2009[17]\end{array}$ & Japan & $\mathrm{JPHC}$ & 12.6 & $49,566 \mathrm{M} ; 54,874 \mathrm{~W}$ & $40-69$ & $\begin{array}{l}52.5 \%(M) \\
6.7 \%(\mathrm{~W})\end{array}$ & $206(79.6 \%)$ \\
\hline Ros, 2011 [21] & $\begin{array}{l}5 \text { European } \\
\text { countries }\end{array}$ & EPIC & 9.3 & $67,914 \mathrm{M} ; 165,322 \mathrm{~W}$ & $\begin{array}{l}53.7(\mathrm{M}) \\
52.9(\mathrm{~W})\end{array}$ & $23.7 \%$ & $513(50.5 \%)$ \\
\hline $\begin{array}{l}\text { Hashibe, } 2015 \\
\text { [31] }\end{array}$ & US & PLCO & 14.0 & 97,334 M\&W & $55-74$ & $9.1 \%$ & 398 (NA) \\
\hline $\begin{array}{l}\text { Loftfield, } 2017 \\
\text { [6] }\end{array}$ & US & $\begin{array}{l}\text { NIH-AARP Diet and } \\
\text { Health }\end{array}$ & 15.5 & $469,047 \mathrm{M} \& W$ & $50-71$ & $14.2 \%$ & $6012(84.6 \%)$ \\
\hline $\begin{array}{l}\text { Sugiyama, } \\
2017 \text { [17] }\end{array}$ & Japan & MCS; OCS & $\begin{array}{l}17.6(\mathrm{MCS}) ; 13.3 \\
(\mathrm{OCS})\end{array}$ & 73,346 M\&W & $\begin{array}{l}40-64(\mathrm{MCS}) ; 40- \\
79 \text { (OCS) }\end{array}$ & $35.3 \%$ & $274(73.7 \%)$ \\
\hline $\begin{array}{l}\text { Gapstur, } 2017 \\
{[5]}\end{array}$ & US & CPS- $\|$ & 23.4 & $\begin{array}{l}\text { 696,391 M\&W } \\
\text { nonsmokers }\end{array}$ & 28-94 & $0 \%$ & $\begin{array}{l}1789 \mathrm{BCa} \\
\text { death (NA) }\end{array}$ \\
\hline Lukic, 2018 [8] & $\begin{array}{l}\text { Norway and } \\
\text { Sweden }\end{array}$ & NOWAC; NSHDS & 13.6 & 193,439 M\&W & $25-74$ & $24.8 \%$ & 479 (59.7\%) \\
\hline $\begin{array}{l}\text { Hashemian, } \\
2019 \text { [9] }\end{array}$ & Finland & ATBC & 17.6 & 26,841 male smokers & 57.2 & $100 \%$ & 835 (100\%) \\
\hline
\end{tabular}

Abbreviations: AHS Adventist Health Study, ATBC Alpha-Tocopherol, Beta-Carotene Cancer Prevention, BCa bladder cancer, CPS Cancer Prevention Study, CVD cardiovascular disease, EPIC European Prospective Investigation into Cancer and Nutrition Study, HPFS Health Professionals Follow-up Study, IWHS lowa Women's Health Study, JPHC Japan Public Health Center, LSS Life-Span Study, M men, MCS Miyagi Cohort Study, NA not available, NCS Netherlands Cohort Study, NIH-AARP National Institutes of Health-American Association of Retired Persons, NOWAC Norwegian Women and Cancer, NSHDS Northern Sweden Health and Disease Study, OCS Ohsaki Cohort Study, PLCO Prostate, Lung, Colorectal, and Ovarian, W women 
Table 2 Reported results and statistical adjustments in the prospective studies included in the meta-analysis

\begin{tabular}{|c|c|c|c|c|}
\hline $\begin{array}{l}\text { Author, } \\
\text { years }\end{array}$ & $\begin{array}{l}\text { Consumption, highest vs. } \\
\text { lowest }\end{array}$ & $\begin{array}{l}\text { RR }(95 \% \mathrm{Cl}) \text {, } \\
\text { highest vs. } \\
\text { lowest }\end{array}$ & Covariate adjustment & Smoking adjustment \\
\hline $\begin{array}{l}\text { Jacobsen, } \\
1986[19]\end{array}$ & $\geq 7$ vs. $\leq 2 \mathrm{cups} / \mathrm{d}$ & $\begin{array}{l}0.99(0.53-1.86) \\
0.98(0.47- \\
2.03)(\mathrm{M})\end{array}$ & $\begin{array}{l}\text { Age, sex, residence, and } \\
\text { smoking (for } M \text { only) }\end{array}$ & $\begin{array}{l}\text { Never, former, current (1-9, 10-19, } \geq 20 \text { cig/d) } \\
\text { (for M only) }\end{array}$ \\
\hline $\begin{array}{l}\text { Mills, } 1991 \\
{[26]}\end{array}$ & $\geq 2$ cups/d vs. never & $\begin{array}{l}1.99(0.91-4.34) \\
2.03(0.70- \\
5.87)(\mathrm{NS}) \\
1.14(0.46- \\
2.80)(\mathrm{FS} / \mathrm{CS})\end{array}$ & Age, sex, and smoking & Never, former, current \\
\hline $\begin{array}{l}\text { Chyou, } 1993 \\
{[27]}\end{array}$ & $\geq 5$ vs. $\leq 1$ times/wk & $2.07(0.84-5.12)$ & Age and smoking & Pack-years $(0,>0-30,>30)$ \\
\hline $\begin{array}{l}\text { Stensvold, } \\
1994[15]\end{array}$ & $\geq 7$ vs. $\leq 2 \mathrm{cups} / \mathrm{d}$ & $\begin{array}{l}1.50(0.45- \\
5.02)(\mathrm{M}) \\
2.40(0.28- \\
20.5)(\mathrm{W})\end{array}$ & $\begin{array}{l}\text { Age, residence, } \\
\text { and smoking }\end{array}$ & Cig/d (continuous) \\
\hline $\begin{array}{l}\text { Michaud, } \\
1999[28]\end{array}$ & $\geq 4 \mathrm{cups} / \mathrm{d}$ vs. $<1 \mathrm{cup} / \mathrm{mo}$ & $0.79(0.48-1.30)$ & $\begin{array}{l}\text { Age, region, energy intake, } \\
\text { fruit and vegetable intake, } \\
\text { and smoking }\end{array}$ & $\begin{array}{l}\text { Smoking status (smoker, nonsmoker) } \\
\text { and pack-years ( } 6 \text { categories) }\end{array}$ \\
\hline $\begin{array}{l}\text { Nagano, } \\
2000[29]\end{array}$ & $\geq 5$ vs. 0 times/wk & $0.90(0.52-1.56)$ & $\begin{array}{l}\text { Age, sex, radiation dose, } \\
\text { education, BMI, calendar } \\
\text { time, and smoking }\end{array}$ & Never, former, current $(\leq 20,>20$ cig/d) \\
\hline $\begin{array}{l}\text { Zeegers, } \\
2001[16]\end{array}$ & $\geq 7(\mathrm{M}) / \geq 5(\mathrm{~W})$ vs. $<2 \mathrm{cups} / \mathrm{d}$ & $\begin{array}{l}1.36(0.82- \\
2.04)(\mathrm{M}) \\
0.32(0.15- \\
0.68)(\mathrm{W})\end{array}$ & $\begin{array}{l}\text { Age, tea consumption, } \\
\text { and smoking }\end{array}$ & $\begin{array}{l}\text { Cig/d (continuous), years of smoking } \\
\text { (continuous) }\end{array}$ \\
\hline $\begin{array}{l}\text { Tripathi, } \\
2002[30]\end{array}$ & $\geq 4$ cups/d vs. $<1 \mathrm{cup} / \mathrm{mo}$ & $1.59(0.95-2.68)$ & Age & None \\
\hline $\begin{array}{l}\text { Kurahashi, } \\
2019[17]\end{array}$ & $\begin{array}{l}\geq 3(\mathrm{M}) / \geq 1(\mathrm{~W}) \mathrm{cup} / \mathrm{d} \text { vs. almost } \\
\text { never }\end{array}$ & $\begin{array}{l}1.37(0.75- \\
2.51)(\mathrm{M}) \\
0.55(0.23- \\
1.33)(\mathrm{W}) \\
2.48(0.88- \\
7.05)(\mathrm{NS})(\mathrm{M}) \\
2.09(0.96- \\
4.54)(\mathrm{FS})(\mathrm{M}) \\
2.24(1.21- \\
4.16)(\mathrm{NS} / \mathrm{FS})(\mathrm{M}) \\
1.13(0.65- \\
1.97)(\mathrm{CS})(\mathrm{M})\end{array}$ & $\begin{array}{l}\text { Age, area, alcohol, green } \\
\text { tea consumption, and } \\
\text { smoking }\end{array}$ & Never, former, current ( $<25, \geq 25$ pack-years) \\
\hline $\begin{array}{l}\text { Ros, } 2011 \\
{[21]}\end{array}$ & $\begin{array}{l}\geq 875(\mathrm{M}) / 500(\mathrm{~W}) \mathrm{ml} / \mathrm{d} \text { vs. }< \\
429(\mathrm{M}) / 250(\mathrm{~W}) \mathrm{ml} / \mathrm{d}\end{array}$ & $1.11(0.85-1.43)$ & $\begin{array}{l}\text { Age, sex, center, energy } \\
\text { intake, and smoking. }\end{array}$ & $\begin{array}{l}\text { Smoking status (never, former and current), } \\
\text { duration (continuous), and lifetime intensity } \\
\text { (continuous) }\end{array}$ \\
\hline $\begin{array}{l}\text { Hashibe, } \\
2015[31]\end{array}$ & $\geq 2$ vs. $<1 \mathrm{cup} / \mathrm{d}$ & $1.08(0.85-1.39)$ & $\begin{array}{l}\text { Age, sex, race, education, } \\
\text { and smoking }\end{array}$ & $\begin{array}{l}\text { Smoking status (never, former, current), } \\
\text { frequency }(1-10,11-20,21-30,>30 \mathrm{cig} / \mathrm{d}) \text {, } \\
\text { duration }(1-10,11-20,>20 \mathrm{yr}) \text {, years since quitting } \\
(>0-2,3-5,6-10,11-20,>20 \mathrm{yr}) \text {. }\end{array}$ \\
\hline $\begin{array}{l}\text { Loftfield, } \\
2017 \text { [6] }\end{array}$ & $\geq 4$ cup/d vs. none & $\begin{array}{l}1.18(1.05-1.33) \\
1.25(1.09- \\
1.43)(\mathrm{M}) \\
0.97(0.74- \\
1.25)(\mathrm{W}) \\
0.87(0.65- \\
1.17)(\mathrm{NS}) \\
1.23(1.04- \\
1.33)(\mathrm{FS}) \\
1.32(0.95- \\
1.81)(\mathrm{CS})\end{array}$ & $\begin{array}{l}\text { Age, sex, race/ethnicity, } \\
\text { BMI, education, reported } \\
\text { health status, fruit intake, } \\
\text { vegetable intake, } \\
\text { supplement use, physical } \\
\text { activity, diabetes, family } \\
\text { history of cancer, and } \\
\text { smoking }\end{array}$ & $\begin{array}{l}\text { Pipes or cigars (ever, never), smoking frequency } \\
(1-10,11-20,21-30,31-40,41-60, \geq 60 \mathrm{cig} / \mathrm{d}) \text {, } \\
\text { years since quitting }(\geq 1-4,5-9, \geq 10 \mathrm{yr})\end{array}$ \\
\hline $\begin{array}{l}\text { Sugiyama, } \\
2017[7]\end{array}$ & $\geq 3$ cup/d vs. none & $\begin{array}{l}0.56(0.32-0.99) \\
0.57(0.31- \\
1.07)(\mathrm{M}) \\
0.44(0.10-\end{array}$ & $\begin{array}{l}\text { Age, sex, BMI, hypertension, } \\
\text { diabetes, Ml, stroke, job status, } \\
\text { education, alcohol, green tea } \\
\text { consumption, walking, and }\end{array}$ & Never, former, current $(<20, \geq 20 \mathrm{cig} / \mathrm{d}$ ) \\
\hline
\end{tabular}


Table 2 Reported results and statistical adjustments in the prospective studies included in the meta-analysis (Continued)

\begin{tabular}{|c|c|c|c|c|}
\hline $\begin{array}{l}\text { Author, } \\
\text { years }\end{array}$ & $\begin{array}{l}\text { Consumption, highest vs. } \\
\text { lowest }\end{array}$ & $\begin{array}{l}\mathrm{RR}(95 \% \mathrm{Cl}) \text {, } \\
\text { highest vs. } \\
\text { lowest }\end{array}$ & Covariate adjustment & Smoking adjustment \\
\hline & & $\begin{array}{l}1.97)(\mathrm{W}) \\
0.62(0.14- \\
2.72)(\mathrm{NS}) \\
0.61(0.32- \\
1.17)(\mathrm{FS} / \mathrm{CS})\end{array}$ & smoking & \\
\hline $\begin{array}{l}\text { Gapstur, } \\
2017[5]\end{array}$ & $\geq 6$ cup/d vs. never & $\begin{array}{l}0.89(0.73- \\
1.09)(\mathrm{NS} / \mathrm{FS}) \\
0.80(0.57- \\
1.12)(\mathrm{NS}) \\
0.97(0.74- \\
1.27)(\mathrm{FS})\end{array}$ & $\begin{array}{l}\text { Age, sex, race, marital status, } \\
\text { education, alcohol consumption, } \\
\text { BMI, physical activity, } \\
\text { family history of cancer, } \\
\text { red and processed meat intake, } \\
\text { vegetable intake, tea } \\
\text { consumption, and smoking. }\end{array}$ & $\begin{array}{l}\text { Years since quitting }(<10,10-<20, \geq 20 \mathrm{yr}) \\
\text { and cig/d }(<20,20-29, \geq 30)\end{array}$ \\
\hline $\begin{array}{l}\text { Lukic, } \\
2018[8]\end{array}$ & $\geq 4$ vs. $<1 \mathrm{cup} / \mathrm{d}$ & $\begin{array}{l}1.34(0.94-1.90) \\
1.23(0.78- \\
1.95)(\mathrm{M}) \\
1.46(0.84- \\
2.51)(\mathrm{W}) \\
1.87(1.01- \\
3.45)(\mathrm{NS}) \\
1.18(0.77- \\
1.81)(\mathrm{FS} / \mathrm{CS})\end{array}$ & Age, sex, and smoking & Never, former, current \\
\hline $\begin{array}{l}\text { Hashemian, } \\
2019[9]\end{array}$ & $\geq 4$ vs. $<1 \mathrm{cup} / \mathrm{d}$ & $\begin{array}{l}1.10(0.81- \\
1.49)(\mathrm{CS})\end{array}$ & $\begin{array}{l}\text { Age, education, alcohol, } \\
\text { diabetes, physical activity, } \\
\text { fruit intake, vegetable intake, } \\
\text { tea consumption, and smoking }\end{array}$ & Smoking years (continuous), cig/d (continuous) \\
\hline
\end{tabular}

Abbreviations: $B M I$ body mass index, cig cigarettes, CS current smoker, FS former smoker, $M$ men, $M I$ myocardial infarction, mo month, NS never smoker, $W$ women, wk, week

a $95 \% \mathrm{Cls}$ were calculated using raw data

${ }^{\mathrm{b}}$ Data were rescaled by using the lowest consumption group as the reference

observed in both high-vs-low $\left(I^{2}=30.4 \%\right)$ and dose-response analyses $\left(I^{2}=56.3 \%\right)$. There was no evidence of a nonlinear association between coffee consumption and risk of bladder cancer $\left(P_{\text {nonlinearity }}=0.65\right)$.

\section{Subgroup and sensitivity analyses}

Results of subgroup analyses according various predefined study and population characteristics are shown in Table 3. For most of these subgroup populations, coffee consumption was not significantly associated with risk of bladder cancer, either in the high-vs-low or in the dose-response analyses. Number of bladder cancer cases $(P$ difference $=0.06$ and 0.04 in the high-vs-low and dose-response analyses, respectively) in addition to the degree of adjustment for smoking $(P$ difference $=0.04$ and 0.06 in the high-vs-low and doseresponse analyses, respectively) were suggested as potential sources of heterogeneity. A stronger positive association between coffee consumption and risk of bladder cancer was observed for studies with smaller $(<200)$ number of cases $\left(\mathrm{RR}\right.$ high-vs-low $=1.38,95 \%$ CI: $1.05-1.81 ; \mathrm{RR}_{1} \mathrm{cup} / \mathrm{d}=1.12$, 95\% CI: 1.04-1.21), and for those with poorer adjustment for smoking (i.e. without adjustment, or with adjustment for smoking status only) ( $R R$ high-vs-low $=1.48,95 \%$ CI: $1.14-$ 1.93; RR ${ }_{1 \mathrm{cup} / \mathrm{d}}=1.08,95 \%$ CI: $\left.1.02-1.15\right)$. Adjustment for tea consumption also emerged as a potential source heterogeneity in the high-vs-low analysis $(P$ difference $=0.042)$ but not in the dose-response analysis $(P$ difference $=0.26$ ).

Combined results from six prospective studies $[5-8,17,26]$ suggested that coffee consumption was not associated with bladder cancer risk among never smokers ( $R R$ high-vs-low $=$ 1.15, 95\% CI: 0.79-1.67; RR 1 cup/d $=1.02,95 \%$ CI: 0.95-1.09). The lack of association persisted in other smoking categories, although dose-response (but not high-vs-low) analysis suggested modestly increased risk among current smokers. Results of the meta-analysis were similar after excluding one study [5] looking at cancer mortality and another study [9] fully consisting of male smokers (Table 3 ).

\section{Publication bias}

For both high-vs-low and dose-response analyses, there was no evidence of publication bias according to results of Begg test or Egger test (all $P$ values $>0.40$ ).

\section{Discussion}

In this meta-analysis of 16 prospective studies including over 2.1 million participants and 11,000 bladder cancer cases, coffee consumption was not associated with risk of bladder. The lack of association persisted in the strata defined by various study characteristics including sex and participants' smoking status. Meta-regression analyses identified the number cases and the degree of 


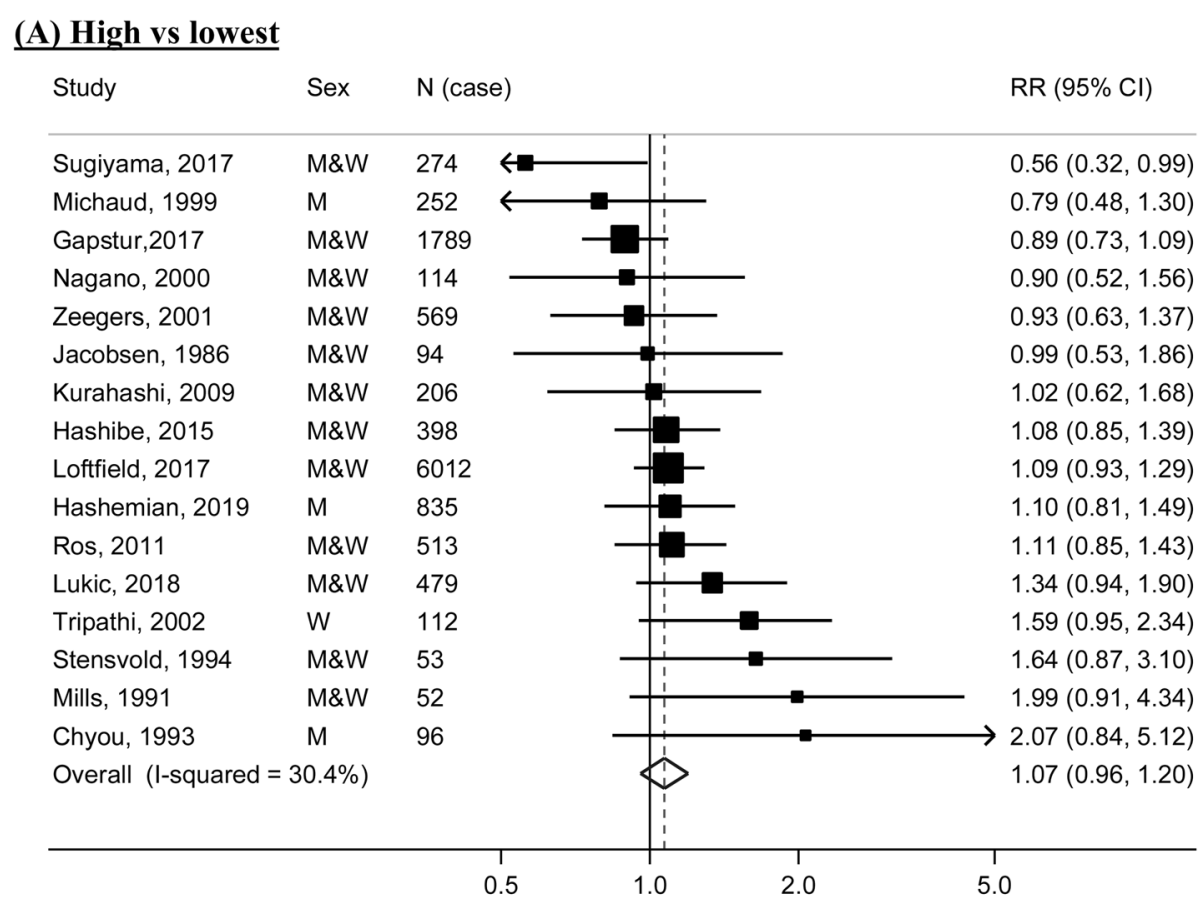

\section{(B) Each 1 cup/d increment}

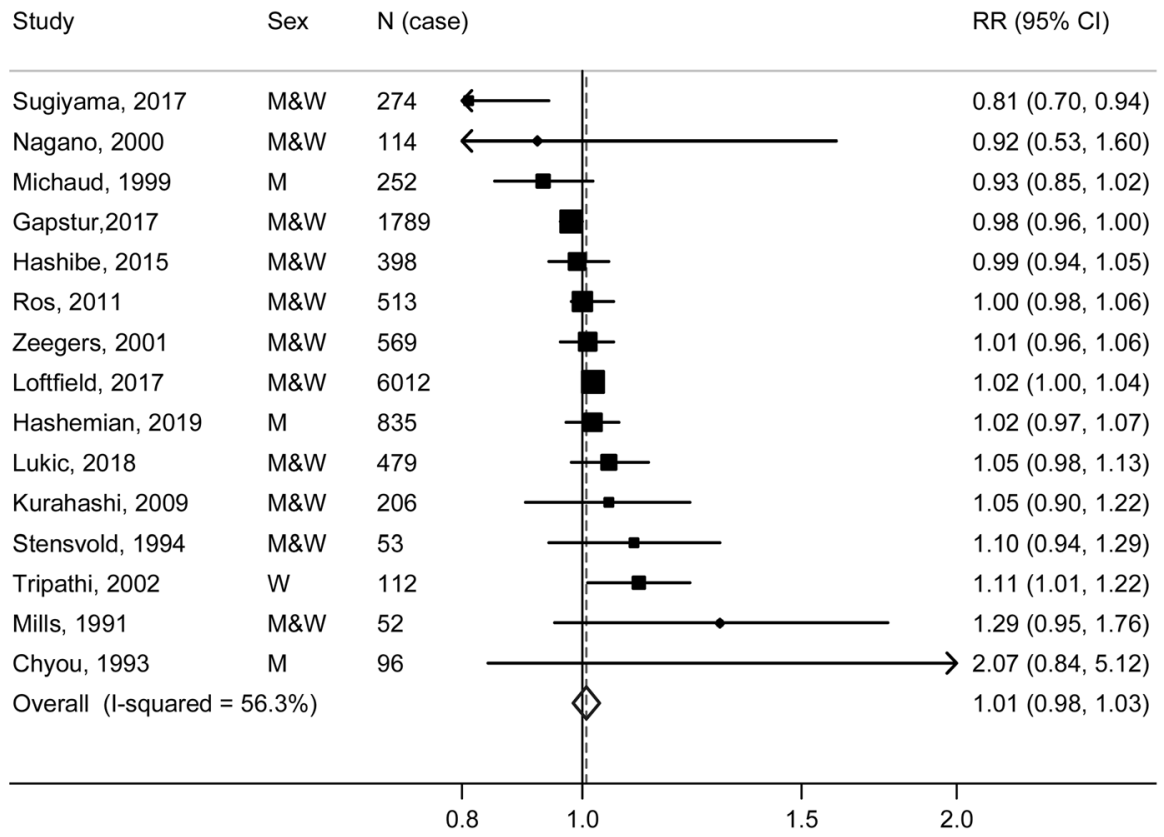

Fig. 2 Forest plots for the meta-analysis of coffee consumption and risk of bladder cancer. a highest vs. lowest analysis; $\mathbf{b}$ dose-response analysis of 1 cup/d increment. $M$, men; W, women

adjustment for smoking as potential sources of heterogeneity. Coffee consumption was associated with increased risk of bladder cancer among studies with fewer cancer cases and among those with poorer adjustment for smoking, indicating that some direct associations observed in individual studies may be a results of residual confounding by smoking.
For studies of coffee consumption and smoking-related cancers including bladder cancer, potential confounding by smoking merits particular attention. Observationally [5, 6] and genetically [32], heavier coffee consumers are more likely to be smokers. In a large cohort of US population [6], only 5.3\% of coffee non-drinkers were current smokers, whereas the 
Table 3 Subgroup and sensitivity analyses

\begin{tabular}{|c|c|c|c|c|c|c|c|c|}
\hline & \multicolumn{4}{|c|}{ Highest vs. lowest consumption } & \multicolumn{4}{|c|}{ Each 1 cup/d increment } \\
\hline & $\bar{N}$ & RR $(95 \% \mathrm{Cl})$ & $P^{2}(\%)$ & $P$-diff & $\bar{N}$ & RR $(95 \% \mathrm{Cl})$ & $R^{2}(\%)$ & $P$-diff \\
\hline \multicolumn{9}{|l|}{ Subgroup analyses } \\
\hline \multicolumn{9}{|l|}{ Geographic region } \\
\hline US & 7 & $1.10(0.92-1.32)$ & 50.4 & Ref. & 7 & $1.01(0.97-1.04)$ & 70.0 & Ref. \\
\hline Europe & 6 & $1.13(0.98-1.31)$ & 0 & 0.60 & 5 & $1.02(0.99-1.04)$ & 0 & 0.55 \\
\hline Japan & 3 & $0.81(0.57-1.16)$ & 23.1 & 0.23 & 3 & $0.92(0.75-1.14)$ & 65.3 & 0.30 \\
\hline \multicolumn{9}{|l|}{ Duration of follow-up } \\
\hline$\geq 10 \mathrm{yr}$ & 12 & $1.08(0.95-1.23)$ & 35.9 & & 11 & $1.01(0.98-1.04)$ & 62.6 & \\
\hline$<10 \mathrm{yr}$ & 4 & $1.04(0.81-1.35)$ & 31.4 & 0.86 & 4 & $1.00(0.95-1.04)$ & 42.3 & 0.67 \\
\hline \multicolumn{9}{|l|}{ No. of participants } \\
\hline$\geq 50,000$ & 8 & $1.02(0.91-1.15)$ & 28.9 & & 8 & $1.00(0.98-1.03)$ & 61.1 & \\
\hline$<50,000$ & 8 & $1.21(0.96-1.51)$ & 28.9 & 0.26 & 7 & $1.04(0.97-1.13)$ & 52.0 & 0.67 \\
\hline \multicolumn{9}{|l|}{ Sex } \\
\hline Men & 10 & $1.16(1.00-1.35)$ & 21.5 & & 9 & $1.01(0.98-1.05)$ & 42.0 & \\
\hline Women & 7 & $0.90(0.59-1.37)$ & 63.6 & 0.37 & 6 & $0.98(0.89-1.08)$ & 56.0 & 0.76 \\
\hline \multicolumn{9}{|l|}{ No. of cases } \\
\hline$\geq 200$ & 10 & $1.02(0.92-1.13)$ & 19.0 & & 10 & $1.00(0.98-1.02)$ & 57.3 & \\
\hline$<200$ & 6 & $1.38(1.05-1.81)$ & 12.0 & 0.064 & 5 & $1.12(1.04-1.21)$ & 0 & 0.037 \\
\hline \multicolumn{9}{|l|}{$\%$ Male cases } \\
\hline$\geq 75 \%$ & 6 & $1.10(0.95-1.26)$ & 4.1 & & 6 & $1.02(0.94-1.10)$ & 69.3 & \\
\hline$<75 \%$ & 9 & $1.10(0.92-1.30)$ & 37.6 & 0.98 & 7 & $1.01(0.99-1.04)$ & 18.5 & 0.87 \\
\hline \multicolumn{9}{|l|}{ \% Current smoker } \\
\hline$\geq 25 \%$ & 8 & $1.02(0.82-1.25)$ & 26.3 & & 7 & $1.00(0.94-1.07)$ & 52.6 & \\
\hline$<25 \%$ & 8 & $1.10(0.96-1.26)$ & 40.5 & 0.60 & 8 & $1.01(0.98-1.03)$ & 63.6 & 0.98 \\
\hline \multicolumn{9}{|l|}{ Smoking status } \\
\hline Never & 6 & $1.15(0.79-1.67)$ & 56.5 & Ref. & 6 & $1.02(0.95-1.09)$ & 58.0 & Ref. \\
\hline Former & 3 & $1.18(0.92-1.52)$ & 55.5 & 0.80 & 3 & $1.01(0.97-1.05)$ & 69.7 & 0.99 \\
\hline Never/former & 6 & $1.20(0.93-1.56)$ & 61.7 & 0.71 & 6 & $1.03(0.98-1.08)$ & 76.3 & 0.80 \\
\hline Current & 3 & $1.19(0.97-1.46)$ & 0 & 0.72 & 3 & $1.04(1.01-1.07)$ & 0 & 0.50 \\
\hline Former/current & 4 & $1.12(0.88-1.43)$ & 33.3 & 0.81 & 4 & $1.01(0.95-1.07)$ & 38.4 & 0.73 \\
\hline \multicolumn{9}{|l|}{ Statistical adjustment } \\
\hline Smoking ${ }^{a}$ & & & & & & & & \\
\hline
\end{tabular}

Statistical adjustment

Smoking $^{\mathrm{a}}$

$\begin{array}{llll}\text { Poorer } & 3 & 1.48(1.14-1.93) \\ \text { Moderate } & 8 & 1.00(0.80-1.26) & 0 \\ \text { Better } & 5 & 1.03(0.93-1.13)\end{array}$


Table 3 Subgroup and sensitivity analyses (Continued)

\begin{tabular}{|c|c|c|c|c|c|c|c|c|c|}
\hline & & & lowest consump & & & Eac & o/d increment & & \\
\hline & & $\bar{N}$ & RR $(95 \% \mathrm{Cl})$ & $R^{2}(\%)$ & $P$-diff & $\bar{N}$ & RR $(95 \% \mathrm{Cl})$ & $P^{2}(\%)$ & $P$-diff \\
\hline Yes & 3 & & $1.02(0.88-1.17)$ & 24.4 & 0.48 & 3 & $1.00(0.97-1.04)$ & 75.7 & 0.90 \\
\hline BMI & & & & & & & & & \\
\hline No & 12 & & $1.15(1.02-1.29)$ & 5.1 & & 12 & $1.02(0.99-1.05)$ & 33.9 & \\
\hline Yes & 4 & & $0.92(0.74-1.14)$ & 52.9 & 0.099 & 3 & $0.98(0.93-1.03)$ & 80.7 & 0.31 \\
\hline Diabetes & & & & & & & & & \\
\hline No & 13 & & $1.10(0.96-1.25)$ & 27.1 & & 12 & $1.01(0.98-1.04)$ & 43.2 & \\
\hline Yes & 3 & & $0.97(0.73-1.29)$ & 60.4 & 0.57 & 3 & $0.99(0.92-1.05)$ & 78.4 & 0.78 \\
\hline Family history of cancer & & & & & & & & & \\
\hline No & 14 & & $1.10(0.96-1.27)$ & 27.9 & & 13 & $1.01(0.98-1.05)$ & 49.9 & \\
\hline Yes & 2 & & $0.99(0.82-1.21)$ & 57.6 & 0.41 & 2 & $1.00(0.96-1.04)$ & 87.0 & 0.74 \\
\hline Energy intake & & & & & & & & & \\
\hline No & 14 & & $1.08(0.96-1.23)$ & 35.3 & & 13 & $1.01(0.99-1.04)$ & 59.0 & \\
\hline Yes & 2 & & $1.00(0.74-1.36)$ & 28.9 & 0.74 & 2 & $0.98(0.91-1.04)$ & 51.3 & 0.43 \\
\hline $\begin{array}{l}\text { Fruit/vegetable } \\
\text { consumption }\end{array}$ & & & & & & & & & \\
\hline No & 12 & & $1.13(0.97-1.33)$ & 32.1 & & 11 & $1.02(0.98-1.06)$ & 51.5 & \\
\hline Yes & 4 & & $1.00(0.88-1.14)$ & 16.5 & 0.29 & 4 & $1.00(0.97-1.03)$ & 72.1 & 0.54 \\
\hline Tea consumption & & & & & & & & & \\
\hline No & 11 & & $1.15(1.02-1.29)$ & 11.4 & & 10 & $1.02(0.99-1.05)$ & 40.8 & \\
\hline Yes & 5 & & $0.92(0.96-1.20)$ & 12.3 & 0.042 & 5 & $0.99(0.96-1.03)$ & 62.1 & 0.26 \\
\hline Sensitivity analysis & & & & & & & & & \\
\hline Excluding 1 study ${ }^{b}$ & & 15 & $1.10(0.98-1.23)$ & 22.4 & & 14 & $1.01(0.99-1.04)$ & 47.0 & \\
\hline Excluding 2 studies ${ }^{c}$ & & 14 & $1.10(0.97-1.25)$ & 27.9 & & 13 & $1.01(0.98-1.04)$ & 51.0 & \\
\hline
\end{tabular}

" $N$ " indicates the number of studies included in the analyses; " $P$-diff" indicates $P$ values for differences between subgroup population (derived using meta-regression analyses)

a Poorer adjustment for smoking: no adjustment for smoking or adjustment for smoking status only; moderate adjustment for smoking: adjustment for smoking status in addition smoking frequency (e.g. cigarettes smoked per day, or pack-years of smoking) either continuously or categorically; better adjustment for smoking: adjustment for smoking status, smoking frequency, in addition to smoking duration or lifetime smoking intensity. One study (Gaspstur, 2017) that reported results only for nonsmokers (never and former smokers) and adjusted for smoking history for former smokers was included in the "better" group. Another study (Hashemian, 2019) that included totally current smokers and adjusted for both smoking frequency and duration was included in the "moderate group" (this study further examined coffee-bladder cancer association by smoking frequency and did not find group differences in the association)

${ }^{b}$ Excluding one study (Gaspstur, 2017) in which the study outcome was bladder cancer mortality, and all analyzed participants were never or former smokers

' In addition to the above-mentioned study (Gaspstur, 2017), further excluding another study (Hashemian, 2019) in which all participants were current smokers

corresponding data in those consuming $\geq 4 \mathrm{cups} / \mathrm{d}$ of coffee was $25.8 \%$. For current smokers, coffee consumption tends to be associated with larger smoking amount [6] and longer smoking duration [31]; for former smokers, habitual coffee drinkers are likely to have been quitting smoking for shorter duration [6]. All these coffee-related smoking features are associated with increased risk of bladder cancer when comparing with never smoking [33]. Thus, adjustment for smoking among individual studies needs to be carefully evaluated in meta-analyses of coffee consumption and smoking-related cancer.

Several previous meta-analysis [10-13] of published case-control and cohort studies suggested increased risk of bladder cancer associated with higher consumption of coffee. However, the overall positive association was totally driven by case-control data, and all of these metaanalyses showed no significant coffee-bladder cancer association in the cohort-only analyses (including nine [13] or fewer cohorts [10-12]), which is concordant with findings of the current meta-analysis. Of note, in the 2015 meta-analysis by $\mathrm{Wu}$ et al [12], it was suggested that coffee consumption was substantially associated with a $72 \%$ increased risk of bladder cancer among nonsmokers (never and former smokers). More recently, results from a pooled analysis of 13 case-control studies also suggested a positive association between coffee consumption and risk of bladder cancer in never smokers [34]. Such positive associations in these previous analyses, however, may have been largely or fully driven by residual confounding by smoking history (former smokers relative to never smokers still have increased 
bladder cancer risk [33]) or by serious information biases (e.g. recall bias) inherent in the original case-control studies. Being aware of earlier information that coffee may be carcinogenic for the bladder (e.g. IARC classified coffee as "possibly carcinogenic" to the bladder in 1991 [3]), patients with bladder cancer in the case-control studies may exaggerate the amount of coffee consumed. Evaluating the association among never smoking individuals using prospective data can avoid these biases. In the current meta-analysis, a subgroup analysis pooling data from six prospective studies, four of which were published after the Wu et al' meta-analysis [12], did not support an association between coffee consumption and risk of bladder cancer among never smokers.

Among the complex mixture of hundreds of chemicals contained in coffee, caffeine has been proposed as having anti-cancer properties [35], which raises the question as to whether any inverse association between coffee consumption and bladder cancer was diluted by consumption of decaffeinated coffee. However, consumption of decaffeinated coffee was uncommon during the initiations of the most original studies, such that only a few studies were able to compare two types of coffee for the association with bladder cancer, observing no meaningful differences in the associations [5, 28]. Also, the amount of other potentially anti-carcinogenic compounds in coffee such as the coffee diterpenes cafestol and kahweol [36] my differ by the brewing methods of coffee, with higher amount in unfiltered (including boiled) coffee than in filtered or instant coffee [37]. Two prospective studies $[8,9]$ evaluated both filtered and boiled coffee in relation to bladder cancer risk, and neither find apparent difference in the association according to coffee brewing methods, though additional studies are still needed.

Our meta-analysis has several strengths. The prospective design of original studies eliminated the influence of recall and selection biases on the examined association. The large number of cases enhanced the statistical power and enabled us to detect any modest association between coffee consumption and bladder cancer risk. The robustness of our findings was further supported by the results of various analyses defined according to smoking status and the degree of adjustment for smoking and by the concordance of results from high-vs-low and dose-response meta-analyses. There are also some limitations to this meta-analysis. All primary studies collected information on coffee consumption only once at baseline without updating data, which may have led to regression dilution bias and thus an attenuated association. In addition, whether the association between coffee and bladder cancer may differ by roasting degree of coffee beans or brewing methods for coffee preparation $[8,9]$, or by histological subtypes of bladder cancer [21] cannot be evaluated in the current meta-analysis due to few or no eligible studies available. Finally, the differences in the association of coffee consumption with risk of bladder according to the number of cases or smoking adjustment were only marginally significant. Given the multiple subgroup analyses performed, the possibility of chance findings cannot be excluded and future large prospective studies with careful consideration of potential confounding by smoking are still warranted.

\section{Conclusion}

In summary, findings from this large meta-analysis of prospective studies suggest that coffee consumption was not significantly associated with long-term risk of bladder cancer. Such a null association was similar for men and women, and was confirmed in never smokers. Thus, best evidence available to date does not support the notion that consumption of coffee may increase the risk of bladder cancer.

\section{Supplementary information}

Supplementary information accompanies this paper at https://doi.org/10. 1186/s12986-019-0390-3.

Additional file 1. Literature search strategies in the databases. (DOCX $11 \mathrm{~kb})$

\section{Abbreviations \\ $\mathrm{Cl}$ : Confidence interval; IARC: the International Agency for Research on Cancer; RR: Relative risk}

\section{Acknowledgements}

The authors thank members of the Department of Nephrology, HwaMei Hospital, University of Chinese Academy of Sciences for their warm supports on this work.

\section{Authors' contributions}

Z-WD and G-CC conceived and designed the research; Z-WD and K-DC searched the literature; Z-WD extracted data and results were verified by KDC and F-RL; Z-WD, K-DC and F-RL analyzed data and prepared tables and figures; Z-WD drafted the manuscript; X-BW and G-CC critically reviewed and revised the manuscript and contributed to the discussion; and all authors read and approved the final version of the submitted manuscript.

\section{Funding}

This work was supported by the Medical Scientific Research Foundation of Zhejiang Province, China (2019KY174).

\section{Availability of data and materials}

The datasets used and/or analyzed during the current study are available from the manuscript and the corresponding author on reasonable request.

Ethics approval and consent to participate Not applicable.

Consent for publication

Not applicable.

Competing interests

The authors declare that they have no competing interests. 


\section{Author details}

1Department of Nephrology, HwaMei Hospital, University of Chinese Academy of Sciences, Ningbo 315010, China. ${ }^{2}$ Department of Epidemiology, School of Public Health, Southern Medical University (Guangdong Provincial Key Laboratory of Tropical Disease Research), Guangzhou 510000, China. ${ }^{3}$ Department of Epidemiology and Population Health, Albert Einstein College of Medicine, 1300 Morris Park Avenue, Bronx, NY 10461, USA.

Received: 16 June 2019 Accepted: 6 September 2019

Published online: 13 September 2019

\section{References}

1. Poole R, Kennedy OJ, Roderick P, Fallowfield JA, Hayes PC, Parkes J. Coffee consumption and health: umbrella review of meta-analyses of multiple health outcomes. BMJ. 2017:359:j5024.

2. Alicandro G, Tavani A, La Vecchia C. Coffee and cancer risk: a summary overview. Eur J Cancer Prev. 2017;26(5):424-32.

3. Cancer IAfRo. Coffee, tea, mate, Methylxanthines, and Methyglyoxal. In: IARC Monograph on the Evaluation of Carcinogenic Risk of Chemicals to Humans Suppl; 1991. p. 51.

4. Loomis D, Guyton KZ, Grosse Y, Lauby-Secretan B, El Ghissassi F, Bouvard V, et al. Carcinogenicity of drinking coffee, mate, and very hot beverages. Lancet Oncol. 2016;17(7):877-8

5. Gapstur SM, Anderson RL, Campbell PT, Jacobs EJ, Hartman TJ, Hildebrand JS, et al. Associations of coffee drinking and Cancer mortality in the Cancer prevention study-II. Cancer Epidemiol Biomark Prev. 2017:26(10):1477-86.

6. Loftfield E, Freedman ND, Inoue-Choi M, Graubard BI, Sinha R. A prospective investigation of coffee drinking and bladder Cancer incidence in the United States. Epidemiology. 2017;28(5):685-93.

7. Sugiyama K, Sugawara Y, Tomata Y, Nishino Y, Fukao A, Tsuji I. The association between coffee consumption and bladder cancer incidence in a pooled analysis of the Miyagi cohort study and Ohsaki cohort study. Eur J Cancer Prev. 2017;26(2):125-30.

8. Lukic M, Nilsson LM, Skeie G, Lindahl B, Braaten T. Coffee consumption and risk of rare cancers in Scandinavian countries. Eur J Epidemiol. 2018;33(3): 287-302.

9. Hashemian M, Sinha R, Murphy G, Weinstein SJ, Liao LM, Freedman ND, et al. Coffee and tea drinking and risk of cancer of the urinary tract in male smokers. Ann Epidemiol. 2019;34(June):33-9.

10. Zhou Y, Tian C, Jia C. A dose-response meta-analysis of coffee consumption and bladder cancer. Prev Med. 2012;55(1):14-22

11. Huang TB, Guo ZF, Zhang XL, Zhang XP, Liu H, Geng J, et al. Coffee consumption and urologic cancer risk: a meta-analysis of cohort studies. Int Urol Nephrol. 2014:46(8):1481-93.

12. Wu W, Tong Y, Zhao Q, Yu G, Wei X, Lu Q. Coffee consumption and bladder cancer: a meta-analysis of observational studies. Sci Rep. 2015:5:9051.

13. Hong $X, X u$ Q, Lan $K$, Huang $H$, Zhang $Y$, Chen S, et al. The effect of daily fluid management and beverages consumption on the risk of bladder Cancer: a meta-analysis of observational study. Nutr Cancer. 2018;70(8):1217-27.

14. DerSimonian R, Laird N. Meta-analysis in clinical trials. Control Clin Trials. 1986;7(3):177-88.

15. Stensvold I, Jacobsen BK. Coffee and cancer: a prospective study of 43,000 Norwegian men and women. Cancer Causes Control. 1994;5(5):401-8.

16. Zeegers MP, Dorant E, Goldbohm RA, van den Brandt PA. Are coffee, tea, and total fluid consumption associated with bladder cancer risk? Results from the Netherlands cohort study. Cancer Causes Control. 2001;12(3):231-8.

17. Kurahashi N, Inoue M, Iwasaki M, Sasazuki S, Tsugane S. Japan public health center study G. coffee, green tea, and caffeine consumption and subsequent risk of bladder cancer in relation to smoking status: a prospective study in Japan. Cancer Sci. 2009;100(2):294-1.

18. Hamling J, Lee P, Weitkunat $R$, Ambuhl M. Facilitating meta-analyses by deriving relative effect and precision estimates for alternative comparisons from a set of estimates presented by exposure level or disease category. Stat Med. 2008:27(7):954-70.

19. Jacobsen BK, Bjelke E, Kvale G, Heuch I. Coffee drinking, mortality, and cancer incidence: results from a Norwegian prospective study. J Natl Cancer Inst. 1986;76(5):823-31.

20. Orsini N, Bellocco R, Greenland S. Generalized least squares for trend estimation of summarized dose-respose data. Stata J. 2006;6:40-57.
21. Ros MM, Bas Bueno-de-Mesquita HB, Buchner FL, Aben KK, Kampman E, Egevad $L$, et al. Fluid intake and the risk of urothelial cell carcinomas in the European prospective investigation into Cancer and nutrition (EPIC). Int J Cancer. 2011;128(11):2695-708.

22. Orsini N, Li R, Wolk A, Khudyakov P, Spiegelman D. Meta-analysis for linear and nonlinear dose-response relations: examples, an evaluation of approximations, and software. Am J Epidemiol. 2012;175(1):66-73.

23. Higgins JP, Thompson SG. Quantifying heterogeneity in a meta-analysis. Stat Med. 2002;21(11):1539-58.

24. Egger M, Davey Smith G, Schneider M, Minder C. Bias in meta-analysis detected by a simple, graphical test. BMJ. 1997:315(7109):629-34.

25. Begg CB, Mazumdar M. Operating characteristics of a rank correlation test for publication bias. Biometrics. 1994;50(4):1088-101.

26. Mills PK, Beeson WL, Phillips RL, Fraser GE. Bladder cancer in a low risk population: results from the Adventist health study. Am J Epidemiol. 1991; 133(3):230-9.

27. Chyou PH, Nomura AM, Stemmermann GN. A prospective study of diet, smoking, and lower urinary tract cancer. Ann Epidemiol. 1993;3(3):211-6.

28. Michaud DS, Spiegelman D, Clinton SK, Rimm EB, Curhan GC, Willett WC, et al. Fluid intake and the risk of bladder cancer in men. N Engl J Med. 1999; 340(18):1390-7.

29. Nagano J, Kono S, Preston DL, Moriwaki H, Sharp GB, Koyama K, et al. Bladder-cancer incidence in relation to vegetable and fruit consumption: a prospective study of atomic-bomb survivors. Int J Cancer. 2000;86(1):132-8.

30. Tripathi A, Folsom AR, Anderson KE. lowa Women's health S. risk factors for urinary bladder carcinoma in postmenopausal women. The lowa Women's health study. Cancer. 2002;95(11):2316-23.

31. Hashibe M, Galeone C, Buys SS, Gren L, Boffetta P, Zhang ZF, et al. Coffee, tea, caffeine intake, and the risk of cancer in the PLCO cohort. Br J Cancer. 2015;113(5):809-16.

32. Bjorngaard JH, Nordestgaard AT, Taylor AE, Treur JL, Gabrielsen ME, Munafo $M R$, et al. Heavier smoking increases coffee consumption: findings from a Mendelian randomization analysis. Int J Epidemiol. 2017:46(6):1958-67.

33. van Osch FH, Jochems SH, van Schooten FJ, Bryan RT, Zeegers MP. Quantified relations between exposure to tobacco smoking and bladder cancer risk: a meta-analysis of 89 observational studies. Int J Epidemiol. 2016:45(3):857-70

34. Yu EY, Wesselius A, van Osch F, Stern MC, Jiang X, Kellen E, et al. The association between coffee consumption and bladder cancer in the bladder cancer epidemiology and nutritional determinants (BLEND) international pooled study. Cancer Causes Control. 2019;30(8):859-70.

35. Ludwig IA, Clifford MN, Lean ME, Ashihara H, Crozier A. Coffee: biochemistry and potential impact on health. Food Funct. 2014;5(8):1695-717.

36. Cavin C, Holzhaeuser D, Scharf G, Constable A, Huber WW, Schilter B. Cafestol and kahweol, two coffee specific diterpenes with anticarcinogenic activity. Food Chem Toxicol. 2002:40(8):1155-63.

37. Urgert R, Katan MB. The cholesterol-raising factor from coffee beans. Annu Rev Nutr. 1997:17:305-24.

\section{Publisher's Note}

Springer Nature remains neutral with regard to jurisdictional claims in published maps and institutional affiliations.

Ready to submit your research? Choose BMC and benefit from:

- fast, convenient online submission

- thorough peer review by experienced researchers in your field

- rapid publication on acceptance

- support for research data, including large and complex data types

- gold Open Access which fosters wider collaboration and increased citations

- maximum visibility for your research: over $100 \mathrm{M}$ website views per year

At $\mathrm{BMC}$, research is always in progress.

Learn more biomedcentral.com/submissions 